\title{
El efecto de los gases de relleno sanitario en el crecimiento vegetal
}

\section{The effect of landfill biogas on vegetal growth}

Sanchez-Yañez Juan Manuel ${ }^{1 *}$, Baltierra-Trejo Eduardo ${ }^{1}$, Márquez-Benavides Liliana ${ }^{2}$

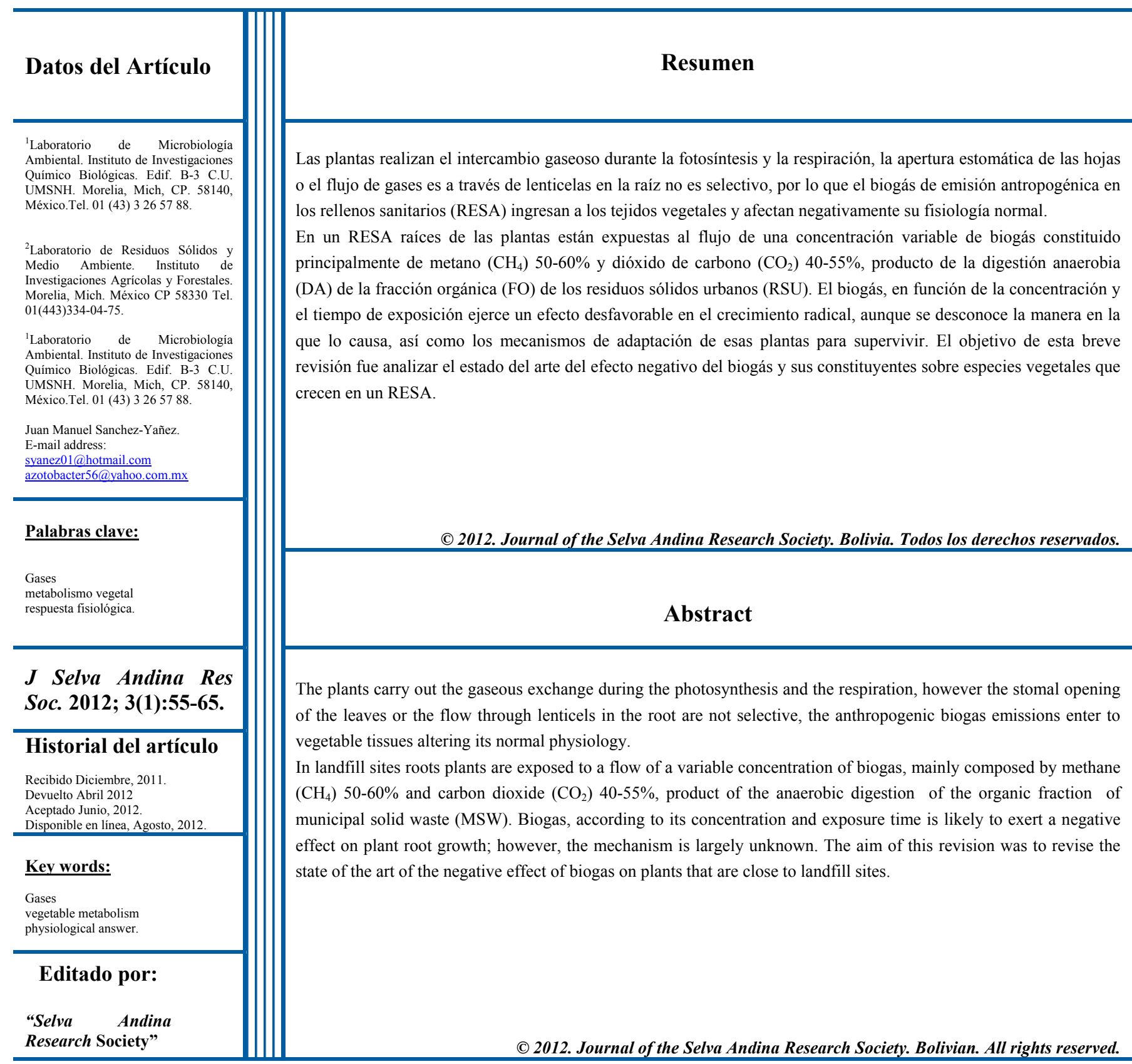




\section{Introducción}

El relleno sanitario (RESA) es el sitio destinado para la disposición final de los residuos sólidos urbanos (RSU) diseñado para minimizar la contaminación del aire, agua y suelo así como los efectos negativos sobre la salud pública (Tchobanoglouset al 2005). Sin embargo, en la práctica los RESA no han logrado ser una solución definitiva que permita controlar la emisión de gases de efecto invernadero lo que contribuye al calentamiento global, ni tampoco para efluentes líquidos como los lixiviados.

Al terminar su vida útil los RESA se rehabilitan como parques, jardines botánicos, áreas de recreación o granjas lo que requiere la introducción de especies vegetales, aunque en ocasiones, las especies sembradas para reforestar tienen dificultades para crecer en estos sitios (Flower et al 1978 citado por Chan et al 1991).

A continuación se describe el proceso por el que el biogás de RESA altera el intercambio gaseoso e inhibe el crecimiento vegetal.

\section{Intercambio de gases en las plantas}

En la fotosíntesis durante la autotrofía el ingreso del dióxido de carbono $\left(\mathrm{CO}_{2}\right)$ permite la síntesis de glucosa y genera oxígeno $\left(\mathrm{O}_{2}\right)$, mientras que en la respiración el $\mathrm{O}_{2}$ oxida azúcares para la liberación de energía con producción de $\mathrm{CO}_{2}$. En la parte aérea vegetal el intercambio gaseoso sucede en los estomas, que normalmente se abren cuando incide la luz en las hojas y se cierran durante la oscuridad. Los gases se mueven en los tejidos vegetales en el espacio aéreo intercelular por debajo de las células del parénquima, para difundir por la pared y membrana celular o por los cauces de aquaporina. Debido a que los gases solo se mueven por difusión, únicamente las capas de células superficiales están vivas mientras que en tallos y raíces las células internas están muertas. En las plantas superiores leñosas se realiza la respiración en la parte subterránea. Sus tallos y raíces maduras están constituidos por capas de células muertas cubiertas con suberina, una cera impermeable a gases, las cuales tienen poros no suberizados o lenticelas que permiten al $\mathrm{O}_{2}$ alcanzar los espacios intercelulares de los tejidos interiores y que el $\mathrm{CO}_{2}$ se difunda a la atmósfera. En plantas herbáceas anuales con tallos verdes se tienen estomas en lugar de lenticelas para el intercambio gaseoso (Salisbury \& Ross 2005).

La concentración de $\mathrm{O}_{2}$ es el principal factor limitante en la respiración radical, la que decrece al disminuir el nivel de $\mathrm{O}_{2}$ (Azcón et al 2001). En suelos inundados la respiración de las raíces de plantas y microorganismos aerobios del suelo consume el $\mathrm{O}_{2}$ rápidamente, mientras que el reemplazamiento por $\mathrm{O}_{2}$ atmosférico es deficiente, debido a que este gas difunde 10000 veces más lento en el agua que en el aire, lo que ocasiona anoxia en el suelo y en consecuencia daño en las raíces (Gil et al 2007).

Algunas plantas requieren alta concentración de $\mathrm{O}_{2}$ para el crecimiento radical. El algodón (Gossypium herbaceum L.) muere si es menor al $0.5 \% \quad(\mathrm{v} / \mathrm{v})$; el jitomate (Lycopersicum esculentumL.) no crece con menos del 4\%, mientras que la frambuesa (Rubus idaeus L.) y manzana (Malus sp. L.) necesitan un mínimo de 
un $10 \%$ de $\mathrm{O}_{2}$ para su desarrollo normal (Chan et al 1991).

En plantas los síntomas de estrés por asfixia radical son: clorosis, episnatía que es la caída del peciolo, abscisión de hojas, disminución del crecimiento, hipertrofia del tallo, marchitamiento de hojas, ausencia de frutos, daño radical, susceptibilidad al ataque patógenos y según el periodo de tiempo de la anoxia la muerte de la planta (Jackson 2002).

Como resultado de la falta de $\mathrm{O}_{2}$ de suelo, ocurre un cambio de respiración de aeróbica en anaeróbica en las raíces de plantas, esto causa una reducción en la síntesis del trifosfato de adenosina (ATP) necesario para el metabolismo aerobio y se acumulan compuestos tóxicos como etanol, ácido láctico, acetaldehído y cianogénicos. Una acidosis citosólica ocurre en las células debido a la acumulación de ácido láctico en el citoplasma seguido de muerte celular (Jackson 2002). La respuesta radical más característica es la sobreproducción de ácido-1-aminociclopropano-1carboxílico (ACC) precursor del etileno en el tallo, también sucede una reducción de la síntesis de citoquininas y giberelinas con un aumento de la concentración de ácido abscísico en hojas que provoca el cierre estomático (Gil et al 2007).

El transporte de $\mathrm{O}_{2}$ a la raíz es importante debido a que está involucrado en la bioquímica de oxidación de carbohidratos para la obtención de ATP, del ión ferroso $\left(\mathrm{Fe}^{2+}\right)$ a férrico $\left(\mathrm{Fe}^{3+}\right)$, de sulfitos $\left(\mathrm{SO}_{3}{ }^{2-}\right)$ a sulfuro $\left(\mathrm{SO}_{4}{ }^{2-}\right)$ y el amonio $\left(\mathrm{NH}_{4}^{+}\right)$a nitrato $\left(\mathrm{NO}_{3}{ }^{-}\right)$.
Las adaptaciones de la raíz vegetal al estrés por ausencia de $\mathrm{O}_{2}$ son: la formación de raíces adventicias, difusión de $\mathrm{O}_{2}$ procedente de la parte aérea, y el desarrollo de un aeroenquima, que es un espacio de aire intercelular formado por disolución de células del córtex del tallo, por el incremento en la concentración del etileno, seguido de la síntesis de celulosas para la digestión del protoplasma y separación de las paredes celulares. Existe un debate sobre si las adaptaciones fisiológicas y morfológicas de las raíces son una respuesta exclusiva el estrés hídrico y no por la anoxia en el suelo (Azcón et al 2001).

\section{La vegetación expuesta al biogás de relleno sanitario}

Las plantas que crecen en RESA y tiraderos a cielo abierto están expuestas a distintas concentraciones de biogás derivado de la digestión anaerobia (DA) de la fracción orgánica (FO) de los residuos sólidos urbanos (RSU), que está compuesto por metano $\left(\mathrm{CH}_{4}\right)$ con un $50-60 \%, \mathrm{CO}_{2}$ con un $40-55 \%$ además de cantidades menores de otros gases y trazas de compuestos orgánicos volátiles (COV) 0.01-0.6\% (Flower et al 1981, Tchobanoglous et al 2005).

Sin embargo, la generación de biogás no es constante a lo largo del tiempo y dependerá de las condiciones existentes en el RESA, así como de la composición de la FO de los RSU. En países industrializados la composición de la FO es menor que en los que se encuentran en vías de desarrollo lo que se verá reflejado en el volumen, composición y tiempo de generación del biogás (Tabla I). La tasa estimada de producción de biogás para México es de 4 a 10 L/kg de RSU/año, 
en condiciones de DA rápida de 3 a 7 años; y de

0.7 a $1.5 \mathrm{~L} / \mathrm{kg}$ de RSU/año en lenta DA de 20 a 40

años (Rosiles 2000).

Tabla 1 Concentración común de gases en un relleno sanitario estabilizado

\begin{tabular}{|c|c|c|c|c|c|}
\hline Gases & EUA \% & EUA \% & EUA \% & Chile \% & Colombia \% \\
\hline Metano & $30-65$ & $30-65$ & $45-60$ & $55-65$ & $54-70$ \\
\hline Dióxido de carbono & $20-50$ & $20-40$ & $40-60$ & $33-44$ & $27-45$ \\
\hline Nitrógeno & $1-21$ & $5-40$ & $2-5$ & $0.2-5$ & \\
\hline Oxígeno & $1-25$ & $0-5$ & $0.1-1.0$ & $0.1-2$ & \\
\hline Amoniaco & & & $0.1-1.0$ & & \\
\hline Hidrógeno & & $1-3$ & $0-0.2$ & & $0-0.1$ \\
\hline Monóxido de Carbono & & & $0-0.2$ & & \\
\hline Sulfuro de Hidrógeno & & $0-0.01$ & $0-1.0$ & $0.001-0.004$ & $0-0.1$ \\
\hline Argón & & $0-0.4$ & & & \\
\hline Referencia & $\begin{array}{c}\text { Bagchi } 1994 \text {, citado } \\
\text { por Robles } 2008\end{array}$ & Johannessen 1999 & Tchobanoglous et al 2005 & $\begin{array}{c}\text { Valdés \& } \\
\text { González } 2003\end{array}$ & Serrano 2008 \\
\hline
\end{tabular}

Se han identificado un total de 116 COV liberados en los RESA (Tabla II), de ellos los compuestos aromáticos son el grupo dominante con $71.5 \%$, seguido por los alcanos con un $6.8 \%$, de las cetonas con $5.7 \%$, de los ácidos orgánicos con un $5.2 \%$, los aldehídos con un 3.0\%, los esteres con un $1 \%$ y los fenoles con un 1\% (Tassi et al 2009).
La mayoría son fitotóxicos como: el cloruro de vinilo, el benceno, el tolueno, el cloroetano y el cloroformo; su generación depende de la codisposición de residuos peligrosos $\mathrm{y}$ de la formación de lixiviados (Tchobanoglous et al 2005).

Tabla 2 Composición frecuente de compuestos traza en el biogás de RESA

\begin{tabular}{|c|c|c|c|}
\hline Gases & Francia $\left(\mathrm{mg} / \mathrm{m}^{3}\right)$ & Reino Unido $\left(\mathrm{mg} / \mathrm{m}^{3}\right)$ & Estados Unidos $\left(\mathrm{mg} / \mathrm{m}^{3}\right)$ \\
\hline $\begin{array}{c}\text { Aromáticos } \\
\text { Ej. Benceno, etil benceno, tolueno }\end{array}$ & 290.76 & $94-330$ & $280-390$ \\
\hline $\begin{array}{c}\text { Cetonas } \\
\text { Ej. Metil, etil cetona }\end{array}$ & 218.82 & - & 130 \\
\hline $\begin{array}{l}\text { Ësteres } \\
\text { Ej. Estirenos }\end{array}$ & 171.05 & 0.1 & 87 \\
\hline Alcoholes & 146.59 & $6-51$ & - \\
\hline $\begin{array}{c}\text { Alifáticos } \\
\text { Halogenados }\end{array}$ & 193.52 & $302-503$ & - \\
\hline $\begin{array}{l}\text { Ej. Cloruro de metano, cloruro de } \\
\text { vinilo, cloroformo }\end{array}$ & 224.64 & $327-739$ & $1-620$ \\
\hline Referencia & Allen et al 1997 & Jaffrin et al 2003 & Tchobanoglous et al 2005 \\
\hline
\end{tabular}

El biogás generado difunde hacia la atmósfera por diferencia en el gradiente de presión y de la temperatura, mientras que a lo largo circula en la dirección de áreas de menor presión en la denominada migración lateral de biogás (MLB), que depende de la porosidad de la clase del suelo adyacente al RESA (Rivera \& Sarmiento 1994).
Se ha registrado que la concentración de $\mathrm{CH}_{4}$ y $\mathrm{CO}_{2}$ supera el $40 \%$ a $100 \mathrm{~m}$ de distancia del borde del RESA y hasta 5\% de $\mathrm{CH}_{4}$ a $300 \mathrm{~m}$ de su límite (Tchobanoglous et al 2005). 


\section{Materiales y métodos}

Se realizó una revisión bibliográfica y en base de datos de revistas científicas conocer el estado del arte del efecto de los gases que componen el biogás de relleno sanitario en el crecimiento vegetal.

\section{Estudios sobre el efecto del biogás en el crecimiento vegetal}

La emisión de biogás producto de la DA de la FO de los RSU se ha ligado con inhibición de la germinación, crecimiento raquítico y pérdida de la vegetación en RESA (Smith et al 2005), sin embargo los estudios realizados son escasos y se desconoce la manera en la que cada uno de esos gases del biogás alteran el metabolismo vegetal, ya sea a nivel de la raíz o de la parte aérea.

\section{Investigaciones "ex situ" del efecto del biogás sobre plantas}

Smith et al (2005) inyectaron en el suelo un flujo regulado de $100 \mathrm{~L} \mathrm{CH}_{4} / \mathrm{h}$ a profundidad de un metro, durante 20 meses sobre el crecimiento del pasto (Bouteloua sp. B.), de trigo (Triticum aestivum L.) y de frijol (Phaseolus vulgaris L.). Los resultados indicaron que el pasto mostró síntomas de clorosis a los 44 días de exposición; mientras que el trigo y frijol tuvieron clorosis y un pobre crecimiento hasta 4 meses después de iniciar el experimento. Aunque la concentración de gas en las parcelas no fue constante, en el punto de inyección se registró hasta $80 \%$ de $\mathrm{CH}_{4}$, pero al aumentar la distancia de éste, la concentración fue menor y en consecuencia el efecto sobre el crecimiento de las plantas. En este modelo de investigación se expuso a las plantas a concentración extremadamente alta de $\mathrm{CH}_{4}$, sin considerar el efecto de $\mathrm{CO}_{2}$, tampoco se simuló la manera en la que el biogás difunde con un flujo extendido y ascendente desde el suelo a la atmósfera. Franzidis et al (2008) con un modelo de simulación por computadora compararon la concentración del biogás en distintos sitios de un RESA durante 3 años; con base a la porosidad del suelo medida por núcleo densimetría o por compactación del suelo, así como por la pérdida de la cobertura vegetal. Los resultados mostraron que la concentración del biogás aumentó en áreas en las que suelo fue poroso, sitio donde a la vez se registró la mayor pérdida de vegetación. Se concluyó que hubo una relación directa entre el nivel de concentración del biogás y la pérdida de la cobertura vegetal en el RESA. Sin embargo, es amplio el número de factores que pueden causar la muerte de las plantas en un sitio de este tipo como: elemento tóxicos en el suelo, el vire del $\mathrm{pH}$, la erosión con pérdida de minerales básicos para la planta y la compactación del suelo que impide el intercambio de gases y el crecimiento radical (Chan et al 1991).

\section{Estudios "in situ" del efecto del biogás sobre vegetales}

Wong et al (1992) investigaron los factores que afectan la diversidad y distribución de la vegetación en un RESA. Para ello hicieron un análisis mediante transectos en cuadrantes de $1 \mathrm{~m}^{2}$ de plantas herbáceas y arbóreas en sitios de baja emisión de biogás de hasta un $10 \%(\mathrm{v} / \mathrm{v})$ de $\mathrm{CH}_{4}$ comparados con los de alta de $41 \%$ de $\mathrm{CH}_{4}$.

Los resultados del efecto del biogás sobre la vegetación en sitios con baja concentración de biogás mostraron mayor cobertura vegetal, con un 
promedio de seis herbáceas con cobertura del $68.5 \%$ y 12 arbóreas superior al 100\%, mientras que en sitios con alta concentración hubo 6 herbáceas que cubrieron $51.2 \%$ y tres arbóreas con el $7.6 \%$, por lo que reportaron una relación directa entre la concentración de biogás y la cobertura vegetal de las herbáceas y las arbóreas. En esta investigación "in situ" no se estableció el efecto negativo directo en el crecimiento vegetal como consecuencia del nivel de emisión de biogás, ni se reportaron en específico cuales fueron las concentraciones reales del $\mathrm{CO}_{2} \mathrm{y}$ del $\mathrm{O}_{2}$ en el muestreo del biogás, lo que limita la interpretación de los resultados.

Por otro lado, Chan et al (1991) estudiaron el impacto del biogás en las raíces de 10 especies de plantas leñosas subtropicales: acacia (Acacia confusa Merr.), acaciade Constantinopla (Albizzia lebbek L.), aporosa (Aporusa chinensis Champ.), ceiba (Bombax malabaricum L.), castanea (Castanopsis fissa Champ.), árbol del ambar (Liquidambar formosana Hance), laurelito (Litsea glutinosa Lour.), aguacate (Persea breviflora Benth.), pino ellioti (Pinus elliottii Engelm.) y boj cepillo (Lophostemon confertus R.Br.). En un RESA se colocaron a distancia de $5 \mathrm{~m}$ entre sí muestreadores de biogás a $30 \mathrm{~cm}$ de profundidad, para determinar sitios con alta y baja emisión de acuerdo con la concentración del $\mathrm{CH}_{4}$; en cada zona trasplantaron 10 plántulas de un año y medio de edad, cada mes durante un año se midieron las variables respuesta: altura, diámetro basal, número y tamaño de hojas, además de porcentaje de cobertura.

Los resultados señalaron que en las áreas con baja concentración en siete especies vegetales no hubo mortalidad, pero si en el aguacate y en la castanea que fue del $60 \%$, en tanto en áreas con alta 60 concentración, hubo menor crecimiento de la parte aérea y disminución de la cobertura, con una mortalidad de plántulas en la mayoría de las especies superior al $10 \%$, mientras que este efecto negativo fue menor en la aporosa, la castanea, el árbol del ámbar, el laurelito y el aguacate con un 50\%. En la acacia y acacia de Constantinopla no hubo inhibición del crecimiento con evidente tolerancia al biogás comparadas con las otras especies vegetales. En los sitios con alta concentración de biogás se determinó su composición química máxima que fue de: $\mathrm{CH}_{4}$ en $41 \%, \mathrm{CO}_{2}$ en $39 \%$ y $\mathrm{O}_{2}$ en $16.3 \%$, mientras que en los de baja: $\mathrm{CH}_{4}$ del $10 \%, \mathrm{CO}_{2}$ del $4.7 \%$ y $\mathrm{O}_{2}$ del $20.3 \%$. Concluyeron que en los sitios de mayor concentración de biogás fue más evidente la inhibición del crecimiento de las plantas leñosas subtropicales.

Las escasas investigaciones realizadas apoyan que el efecto negativo en el crecimiento de plantas en RESA es causado por la condición de anoxia debido al desplazamiento de $\mathrm{O}_{2}$ y se considera que el $\mathrm{CH}_{4}$ y $\mathrm{CO}_{2}$ son gases inertes a la fisiología vegetal, por lo que el daño podrá ser por el efecto mecánico de la asfixia de la raíz, mientras que no se reportan evidencias de la alteración de una ruta metabólica como la síntesis de pigmentos, la respiración, la obtención de energía o de la alteración morfológica (Chan et al 1991).

\section{Impacto de gases en la fisiología vegetal}

El aire de intercambio aéreo vegetal está compuesto por $78 \%$ nitrógeno $\left(\mathrm{N}_{2}\right), 21 \% \mathrm{O}_{2}$, $0.035 \% \mathrm{CO}_{2}, 0.93 \%$ de argón (Ar) y neón $(\mathrm{Ne})$ (Fernández \& Cosían 1987). La variación en la composición y concentración de gases puede causar un efecto positivo o negativo en el 
crecimiento vegetal. Se ha caracterizado principalmente el efecto fitotóxicos sobre la parte aérea vegetal de gases como dióxido de azufre $\left(\mathrm{SO}_{2}\right)$, dióxido de nitrógeno $\left(\mathrm{NO}_{2}\right)$ y ozono $\left(\mathrm{O}_{3}\right)$ productos de las actividades industriales y la quema de combustibles fósiles, los cuales causan alteración del metabolismo respiratorio $\mathrm{y}$ fotosintético vegetal al interferir en la síntesis de proteínas.

El aire de intercambio radical contiene $78-80 \%$ $\mathrm{N}_{2}, \quad 10-20 \% \quad \mathrm{O}_{2}$ y $0.1-3 \% \quad \mathrm{CO}_{2}$ (Fernández \& Kosian 1987). Los factores que pueden afectar el intercambio gaseoso de las raíces son: la temperatura, el contenido de humedad, la materia orgánica, la textura del suelo, la actividad microbiana, la acción de contaminantes y la composición de la mezcla gases en el aire del suelo.

Los gases que componen el biogás inhiben la germinación y el crecimiento vegetal mediante dos vías: 1) Por el efecto mecánico debido al desplazamiento del $\mathrm{O}_{2}$ disponible en el suelo lo que provoca la asfixia radical; 2) Por el efecto químico a causa de la alteración de rutas metabólicas específicas como síntesis de pigmentos y obtención de energía, así como de estructuras celulares.

A continuación se describe los gases que componen el biogás de RESA producto de la DA de la FO de los RSU y la manera en la que podrían alterar la fisiología vegetal de acuerdo a los reportes existentes en la literatura.

\section{Metano}

El $\mathrm{CH}_{4}$ es un gas incoloro, inodoro, inflamable y poco soluble en agua. Desde el comienzo de la revolución industrial se ha duplicado la concentración de $\mathrm{CH}_{4}$ en la atmósfera de la Tierra de 0.8 a 1.7 ppm. Se considera que las principales fuentes generadoras son la minería del carbón, la producción de combustibles fósiles, la agricultura, la ganadería, los pantanos, los tiraderos a cielo abierto de residuos sólidos y los RESA; el 60\% de las emisiones son antropogénicas con una media de $600 \mathrm{Tg}$ año ${ }^{-1}$. En comparación con el $\mathrm{CO}_{2}$, la concentración de $\mathrm{CH}_{4}$ es menor y permanece en la atmósfera menos tiempo, pero su poder de absorción de la radiación infrarroja es ocho veces mayor (Steven et al 2006)

Las hojas de plantas sometidas a estrés hídrico, rayos UV y altas temperaturas emiten $\mathrm{CH}_{4}$ en el orden de $0.0022 \mathrm{ppm}$, se desconoce su función y la ruta metabólica que lo genera (Tassi et al 2009). Se sugiere que el $\mathrm{CH}_{4}$ exógeno no es fitotóxico en el metabolismo vegetal, sin embargo el $\mathrm{CH}_{4}$ no es un componente estándar del aire del suelo, una parte se genera por la DA de la FO realizada por bacterias metanogénicas, aunque de manera natural aumenta desde $0.1-2 \%$ y en suelos anegados hasta un 14\%. En la naturaleza las bacterias metanotróficas usan el $\mathrm{CH}_{4}$ como fuente de carbono y energía mientras que al mismo tiempo estimulan el crecimiento vegetal al fijar $\mathrm{N}_{2}$ (Arif \& Verstraete 1995), pero si no existe suficiente $\mathrm{O}_{2}$ en el suelo su oxidación es incompleta y se generan compuestos secundarios como el metanol, formol, ácido fórmico o etileno que en concentración mayor al 5\% es tóxico para las raíces de las plantas (Jackson 2002). 
En los RESA el $\mathrm{CH}_{4}$ es el principal componente del biogás, las raíces de las plantas que ahí crecen están expuestas a una elevada concentración que es entre un $45-60 \%$, mientras en las consideradas de menor difusión pueden tener un intervalo de entre $0-40 \%$ (Themelis \& Ulloa 2006).

\section{Dióxido de carbono}

En la fisiología vegetal el requerimiento mínimo de $\mathrm{CO}_{2}$ para la fotosíntesis vegetal es de 0.035$0.05 \%$. Un incremento de $\mathrm{CO}_{2}$ aumenta la tasa fotosintética, con mayor crecimiento $y$ rendimiento. Algunos reportes sugieren que la exposición a $0.15-0.2 \%$ de $\mathrm{CO}_{2}$ es positivo para el crecimiento vegetal, lo que mejora su productividad en un $30 \%$, mientras que superior al $1 \%$ es fitotóxico: causa el cierre de estomas, inhibe la fotosíntesis, la respiración y la transpiración (Lindroth 2010).

Las concentración de $\mathrm{CO}_{2}$ en la rizósfera es de $0.1-2 \%\left(<1500 \mu 1 \mathrm{~L}^{-1}\right)$. La tasa respiratoria de los raíces en suelos forestales varía en un rango 350$1000 \mu \mathrm{L} \mathrm{L}^{-1}$ (Burton \& Pregitzer 2002). Incrementar ligeramente la concentración de $\mathrm{CO}_{2}$ estimula el crecimiento radical por un mecanismo de resistencia al estrés anóxico; pero a mayor nivel del 5\% inhibe la germinación, superior al 6.5\% impide la respiración y el crecimiento; mientras que del $15-20 \%$ es letal para la mayoría de las plantas superiores. Un efecto secundario incluye la acidificación del $\mathrm{pH}$ del suelo al reaccionar con el agua y formar $\mathrm{H}_{2} \mathrm{CO}_{3}$ (Stolwijk \& Thimman 1997, Burton \&Pregitzer 2002).

En el suelo de los RESA la concentración de $\mathrm{CO}_{2}$ "normalmente" es de 40-55\% (Tchobanoglous et 62 al 2005), superior a la capacidad reportada como máxima tolerable para las plantas, mientras que puede ejercer un efecto positivo o negativo en la parte aérea vegetal, ya que tarda en disiparse por ser un gas más denso que el aire.

\section{Monóxido de carbono, sulfuro de hidrógeno, amoniaco y compuestos orgánicos volátiles}

Los gases que están en menor proporción o como trazas en los RESA, también se generan discontinuamente por lo que no se consideran los agentes causales principales de la perdida de vegetación en estos sitios, aunque se ha analizado su efecto tóxico en la parte aérea y en algunos casos en la radical en especies de plantas. Así, se reportan efectos tóxicos a baja concentración, sin embargo los modelos experimentales no simulan la manera en la que sucede en un RESA.

El CO es un componente menor del biogás de RESA, el nivel registrado en el suelo es de $0-0.2 \%$ (Tchobanoglous et al 2005). Las plantas son tolerantes a concentración menor al $0.01 \%$ de $\mathrm{CO}$, lo oxidan a $\mathrm{CO}_{2}$, mientras que mayor cantidad puede ser tóxico hasta mortal al evitar que los tejidos vegetales obtengan el $\mathrm{O}_{2}$ necesario para el metabolismo aerobio. Por ejemplo, el CO inhibe la enzima citocromo oxidasa del ciclo de Krebs (Webster 1994).

En RESA el $\mathrm{H}_{2} \mathrm{~S}$ existe en concentración entre un $0-1 \%$ (Tchobanoglous et al 2005). Este compuesto es fitotóxico a un nivel superior al $0.1 \mu \mathrm{g} \mathrm{L}^{-1} \mathrm{y}$ provoca clorosis, defoliación, menor crecimiento y muerte; en general las plantas jóvenes son más susceptibles al daño. Sin embargo, la baja concentración estimula el crecimiento vegetal 
porque es una fuente de azufre (Kok 1988). Los síntomas en planta por exposición a $\mathrm{H}_{2} \mathrm{~S}$ son clorosis intervenal en hojas, raíces gruesas, poco desarrolladas y de color negro, con muerte del sistema radicular por inhibición de la respiración aeróbica al bloquear la actividad de las metaloenzimas como citocromo oxidasa en las mitocondrias y por generar un exceso de $\mathrm{Fe}$ y $\mathrm{Mn}$ en el citosol. Se reporta que las raíces de plantas desarrollan mecanismos de tolerancia a este ácido inorgánico, así el arroz libera $\mathrm{O}_{2}$ para oxidar el $\mathrm{H}_{2} \mathrm{~S}$ en la rizósfera lo que disminuye su toxicidad (Moore et al 1990).

En un RESA el $\mathrm{NH}_{3}$ se emite en concentración del $0.1-1 \%$ (Tchobanoglous et al 2005), generado por la descomposición de compuestos orgánicos nitrogenados por la actividad microbiana. Los síntomas por exposición en plantas son: daño foliar, alteración del crecimiento, susceptibilidad a sequías, heladas, a insectos y microorganismos patógenos. Bioquímicamente el $\mathrm{NH}_{3}$ inhibe la respiración en las mitocondrias, provoca el adelgazamiento de paredes celulares, síntesis de proteínas, la asimilación de Nitrógeno (N) y causar la acumulación de compuestos fitotóxicos en los tejidos. Sin embargo las plantas tienen una parcial resistencia ya que lo oxidan a $\mathrm{NH}_{4}$, que es menos tóxico y es una fuente de $\mathrm{N}$ para la célula (Granados \& López 2001, Krupa 2003).

Los COV como benceno, cloruro de vinilo, cloroformo, cloroetano son altamente fitotóxicos pero se generan en cantidades traza y discontinua por lo que es poco probable que sean la causa de la perdida de vegetación en RESA. Se reporta que en las plantas causan alteración del metabolismo fotosintético y de las membranas celulares. (Tassi et al 2009). Los gases halogenados son fitotóxicos a baja concentración. Las plantas absorben el gas del aire a través de sus hojas, ahí destruye tejidos vegetales, por oxidación y expulsión del Hidrógeno $\left(\mathrm{H}_{2}\right)$ en los compuestos orgánicos. Esto puede producir síntomas característicos como el blanqueamiento y el amarillamiento de las hojas.

Se reporta que el $\mathrm{CO}, \mathrm{H}_{2} \mathrm{~S}, \mathrm{NH}_{3}$ causan alteración de estructuras celulares, de la fotosíntesis y de la respiración, pero se desconoce si la concentración en la que se generan en los RESA, es suficiente para provocar un efecto negativo en el crecimiento de las plantas.

La existencia escasa de vegetación en los RESA, es un indicativo de que los valores considerados como máximos para la exposición de raíces de plantas a los distintos gases en el suelo están subestimados en la literatura, o que algunas plantas que ahí crecen se han adaptado a esa condición adversa, sin embargo no existe información precisa relacionada específicamente con la repuesta vegetal a la acción toxica de esos compuestos.

\section{Conclusiones}

Debido a la escasa investigación del efecto negativo del biogás en el sistema radical de plantas de un RESA no es posible aún dar una explicación precisa, de cuál es el mecanismo por el que se altera la fisiología y metabolismo de las plantas, por principio a nivel de la raíz, ni cuál de los componentes del biogás es el que tiene mayor impacto negativo en el crecimiento vegetal. La actual evidencia indica que es un estrés causado por el desplazamiento del $\mathrm{O}_{2}$ en el aire del suelo 
por el biogás al difundir desde las zona de la producción durante la DA de la FO de los RSU.

\section{Prospectiva}

Las investigaciones sobre el efecto de exponer las raíces de las plantas al biogás, producto de la DA de la FO de los RSU en los RESA podrían explicar cómo esta mezcla de gases perjudica la fisiología normal de la raíz al alterar de la composición del aire rizósferico, lo que en consecuencia induce adaptaciones en las plantas para enfrentar esa condición atípica.

Es necesario realizar investigación bioquímica e histológica en las raíces vegetales expuestas a altos y bajo niveles de biogás, así como detectar géneros de plantas sensibles tolerantes que podrían ser útiles como bioindicadores de la calidad del aire del suelo en un RESA.

\section{Agradecimientos}

A los Proyectos 2.7 y 5.15 (2010-2011) de la CICUMSNH, Morelia, Mich, México, a la Beca 239180 (2010-2012) para EBT, del Consejo Nacional de Ciencia y Tecnología (CONACYT, México) por el apoyo para la realización de este trabajo.

\section{Literatura citada}

Arif MAS, Verstraete W. Methane dosage to soil and its effect on plant growth. World J Microbiol Biotechnol. 1995; 11:520-35.

Allen MR, Braithwaite A, Hills CC. Trace organic compounds in landfill gas at seven UK waste disposal sites. Environ Sci Technol. 1997;
31:1054-61.

AzcónJ, Talón M, Tadeo F. Fundamentos de fisiología vegetal. "Fisiología de las plantas y Estrés”. España: Mc-Graw-Hill. 2001;481-489 pp.

Bagchi A. Design, construction and monitoring of landfills. $2^{\text {nd }}$ ed. U.S.A: Wiley Interscience; 1994. 57 pp.

Burton AJ, Pregitzer KS. Measurement carbon dioxide concentration does not affect root respiration of nine tree species in the field. Tree physiol. 2002; 22:67-72.

Chan GYS, Wong MH, Whitton BA. Effects of landfill gas on subtropical woody plants. Environ Manage. 1991; 15:411-31.

Fernandez IJ, Kosian PA. Soil air carbon dioxide concentrations in a New England spruce-fir forest. Soil Sci Soc Am J. 1987; 51:261-63.

Flower FB, Gilman EF, Leone IA. Landfill gas, what it does to trees and how its injurious effects may be prevented. J Arboric. 1981; $7: 43-52$.

Flower FB, Leone IA., Gilman EF, Arthur JJ. A study of vegetation problems associated with refuse landfills. EPA. U.S.A. 1978; 600:78-94.

Franzidis JP, Héroux M, Nastev M, Guy C. Lateral migration and offsite surface emission of landfill gas at City of Montreal landfill site. Waste Manage Res. 2008; 26:121-31.

Gil P, Schaffer B, Gutiérrez SM, Li C. Efecto del anegamiento en el estatus hídrico e intercambio gaseoso y biomasa del palto (Persea americana Mill.). Proceedings VI World Avocado Congress. Chile. 2007.

Granados D, López GF. Declinación forestal. Chapingo, Ciencias Forestales y del Ambiente. 2001; 7:5-13. 
Jackson M. Long-distance signaling from roots to shoots assessed: the flooding story. J Exp Bot. 2002; 53:175-81.

Jaffrin A, Bentounes N, Joan AM. Landfill biogas for heating greenhouses and providing carbon dioxide supplement for plant growth. Biosyst Eng. 2003; 86:113-23.

Johannessen LM. (The World Bank). Guidance Note on Recuperation of Landfill Gas from Municipal Solid Waste Landfills. Washington, D.C. USA. 1999.

Kok L. Responses of Sulfur Metabolism in Plants to Atmospheric Hydrogen Sulfide. Phyton. 1988; 29:189-201.

Krupa SV. Effects of atmospheric ammonia $\left(\mathrm{NH}_{3}\right)$ on terrestrial vegetation: a review. Environ Pollut. 2003; 124:179-221.

Lindroth RL. Impacts of elevated atmospheric $\mathrm{CO}_{2}$ and $\mathrm{O}_{3}$ on forests: phytochemistry, trophic interactions, and ecosystem dynamics. Journal of Chemistry Ecology. 2010; 36:2-21.

Moore PA, Attanandana T, Patrick WH. Factors affecting rice growth on acid sulfate soils. Soil Sci Soc Am J. 1990; 54:1651-56.

Rivera G., Sarmiento C. Programa de pruebas de campo para la utilización de biogás del relleno sanitario prados de la montaña. Dirección General de Servicios Urbanos, México D.F. 1994. 8 pp.

Robles F. Generación de biogás y lixiviado en los rellenos sanitarios. México: Instituto Politécnico Nacional; 2008. 75 pp.

Rosiles G. (SEDESOL). Modelo Mexicano del Biogás. México, D.F. 2000. 31 pp.

Salisbury FB, Ross CW. Fisiología Vegetal. México: Iberoamérica: 2005. 71-98, 249-313 p.
Serrano C, Sanabria O. Alternativas de utilización de biogás de rellenos sanitarios en Colombia. Proyecto de Tesis de Maestria. Bogota. 2005. $35 \mathrm{pp}$.

Smith KL, Colls JJ, Steven MD. A facility to investigate effects of elevated soil gas concentration on vegetation. Water Air Soil Pollut. 2005; 161:75-96.

Steven MD, Smith KL, Beardsley MD, Colls JJ. Oxygen and methane depletion in soil affected by leakage of natural gas. Eur J Soil Sci. 2006; 57:800-807.

Stolwijk AJ, Thimann KV. On the uptake of carbon dioxide and bicarbonate by roots, and its influence on growth. Plant Physiol. 1997; 32:513-20.

Tassi F, Montegrossi G, Vaselli O, Liccioli C, Moretti S, Nisi B. Degradation of C2-C15 volatile organic compounds in a landfill cover soil. Sci Total Environ. 2009; 407:4513-25.

Tchobanoglous G, Kreith F. Handbook of Solid Wasted Management. New York: McGrawHill; 2005 Cap.14.

Themelis NJ, Ulloa PA. Methane generation in landfills. RenewEnerg. 2006; 32:1243-57.

Valdés S, González JP. (Banco Mundial/ESMAP/CIDA) "Estudio de Casos de Aprovechamiento Energético de biogás de Rellenos Sanitarios en Chile. 2003. 33 pp.

Webster GC. The Effect of Carbon Monoxide on Respiration in Higher Plants. Plant Physiol.1994; 29:399-400.

Wong MH, Cheung KC, Lan CY. Factors related to the diversity and distribution of soil fauna on Gin Drinkers' Bay landfill, Hong Kong. Waste Manag Res. 1992; 10:423-34. 